Linking academia, business and industry through research

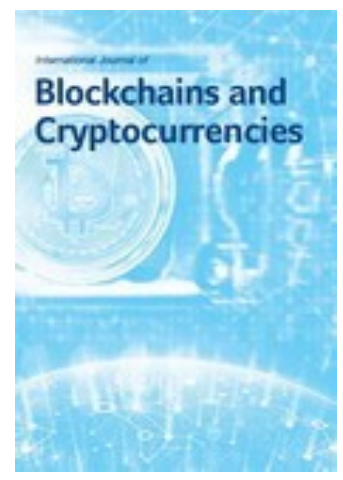

International Journal of Blockchains and Cryptocurrencies

ISSN online: 2516-6433 - ISSN print: 2516-6425

https://www.inderscience.com/jijbc

\title{
Blockchain in accounting: challenges and future prospects
}

Asif Iqbal Baba, Subash Neupane, Fan Wu and Fanta F. Yaroh

DOI: $\underline{10.1504 / / J B C .2021 .117810}$

\section{Article History:}

Received:

Accepted:

29 July 2020

Published online:

2020

25 September 2021 


\title{
Blockchain in accounting: challenges and future prospects
}

\section{Asif Iqbal Baba*, Subash Neupane and Fan $\mathrm{Wu}$}

Department of Computer Science,

Tuskegee University,

Tuskegee, AL, USA

Email: ababa@tuskegee.edu

Email: sneupane2127@tuskegee.edu

Email: fwu@tuskegee.edu

*Corresponding author

\section{Fanta F. Yaroh}

KPMG LLP,

Atlanta, Georgia, USA

Email: fyaroh@kpmg.com

\begin{abstract}
Blockchain technology has the potential to disrupt the existing business models in virtually every sector, including finance, legal, manufacturing, healthcare, government, supply chain, insurance, tourism, agriculture, etc. Because of a myriad of benefits surrounding this technology as such decentralisation, persistency, anonymity, and auditability every business today wants to adopt this technology. Although this technology can be applied to any sector, it is fundamentally considered as accounting technology. It can improve efficiency and significantly change the face of traditional accounting and bookkeeping. The accounting systems of the present are still in their digital infancy in comparison to other industries, largely due to the exceptionally high regulatory requirements concerning the validity and integrity of data and information. In this paper, we will discuss the impact of blockchain technology on the accounting profession, technical, regulatory, and other concerns in detail. We also indicate the directions for the future of blockchain-based accounting.
\end{abstract}

Keywords: blockchain; blockchain architecture; accounting; decentralisation; distributed ledgers.

Reference to this paper should be made as follows: Baba, A.I., Neupane, S., $\mathrm{Wu}$, F. and Yaroh, F.F. (2021) 'Blockchain in accounting: challenges and future prospects', Int. J. Blockchains and Cryptocurrencies, Vol. 2, No. 1, pp.44-67.

Biographical notes: Asif Iqbal Baba received his $\mathrm{PhD}$ in Computer Science at the Aalborg University, Denamrk. Currently, he is an Assistant Professor at the Computer Science Department, Tuekegee University, USA. His research currently focuses on security and privacy issues in IoT, indoor tracking 
and navigation, and big data management. He has published several peer reviewed papers in prestigious journals/conferences such as ACM SIGMOD, ACM SIGSPATIAL, ACM SIGCSE, IEEE MDM, WirelessDays, Elsevier, Inderscience and IGI.

Subash Neupane is a graduate research assistant at the Tuskegee University, Alabama, USA. He is currently pursuing his Master's in Information Systems Security Management. Before joining Tuskegee, he worked at the Tandem Corp, Mulgrave, Victoria, Australia in the capacity of telecom network engineer. He has more than seven years of experience in the IT industry.

Fan $\mathrm{Wu}$ received his $\mathrm{PhD}$ in Computer Science from the Worcester Polytechnic Institute (WPI) in 2008. He is a Professor of Computer Science and the Director of Tuskegee University Center of Academic Excellence in Information Assurance Education. His teaching and research interests lie in primarily in the area of information assurance, software security, mobile security, high performance computing and artificial intelligence. He has led several cybersecurity related projects funded by NSF, DHS, and DoD. He has served as the associate editor of the International Journal of Mobile Devices, Wearable Technology, and Flexible Electronics.

Fanta F. Yaroh is a senior auditor associate at the KPMG US. She completed her Master's in Information Science and Security Management from the Department of Computer Science, Tuskegee University, USA. She received her Bachelor's in Accounting from the Collage of Business and Information Science, Tuskegee University, USA. She has been working in the financial industry for several years now.

\section{Introduction}

Recently blockchain technology has received global recognition across the industries and is seen as a technology that could potentially revolutionise businesses. However, most people heard about blockchain technology probably in the context of cryptocurrencies like Bitcoin and Ethereum (Alladi et al., 2019). The momentum is slowly but surely shifting towards building practical business applications. Besides, the landscape is changing rapidly as blockchain is used in many industries including finance, telecom, healthcare, supply-chain, insurance, education (Han et al., 2018), technology, and government (Casino et al., 2019). While the technology is novel many stakeholders have come to the forefront offering a blockchain platform. The most recent and prominent industry to engage in the blockchain domain is Google (Johnson, 2020).

In a 2019 survey on the blockchain, Deloitte, one of the world's leading financial companies, polled over 1,000 senior executives of the companies across seven different countries (Delliott Insights, 2019). Over 70\% of those senior executives mentioned that they already see a business case in their organisations where blockchain can be used. Some of them have already moved forward with the use of technology. About a third of responding executives (34\%) say their company already has some production blockchain system. In contrast, another $41 \%$ of respondents say they expect their organisations to deploy a blockchain application within the next 12 months. Moreover, 
nearly $40 \%$ of respondents reported that their organisation would invest $\$ 5$ million or more in blockchain technology in the coming year. The report also states that $53 \%$ of respondents went on to say blockchain technology has become a key priority for their institutions in 2019, a ten-point surge over their 2018 survey.

Figure 1 A blockchain snippet (see online version for colours)

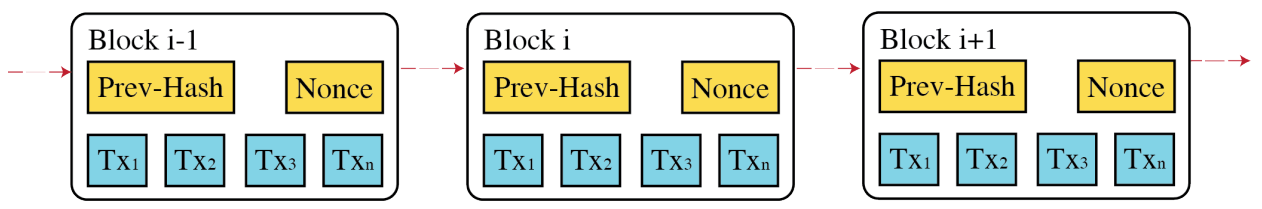

Blockchain, at its core, is a complex, distributed ledger system. The ledger contains connected blocks of transactions for all the participants of the network. Network participants manage and approve transaction updates through a sophisticated consensus protocol. This ensures that a single, agreed version of the transactional record spreads to all network users as part of a permanent record, even without a central authority or a brokerage, such as the financial institution or clearinghouse (Brakeville and Bhargav, 2016). This mechanism generates an impression of 'universal entry bookkeeping', where a single entry is shared identically and permanently with every participant (ICAEW, 2017). Substantial use of cryptography has become a critical feature of blockchain networks, which brings trustworthiness behind all network interactions. While the smart contract, a self-executing script that resides on the blockchain, integrates these concepts and allows for accurate, distributed, programmed workflows (Konstantinos and Micheal, 2016).

In this paper, we examined how this emerging technology could transform accounting and create value for the industry. With the advent of technology, automating business processes is in high demand. In the context of the accounting profession, blockchain can further enhance this industry by reducing the costs of maintaining and reconciling ledgers and providing absolute certainty over the ownership and history of assets. For example, for auditing, blockchain presents one of the greatest opportunities by harnessing big data to make audits more insightful, faster, and efficient.

Some of the key benefits that blockchain brings to accounting are: Efficiency: with blockchain pushing data into and out of the system will be much faster than traditional accounting software. Reliability: with the automation of transactions, forming a chain, smart accounting function will drastically reduce human error. Cost-effectiveness: improved efficiency and error reduction with consequently reduce the cost. Firms will have to bear a cost upfront for initial adoption. Fraud reduction: with blockchain implementation, it is difficult to manipulate the records. Since blockchain is a distributed ledger, making a chain to record means the same change needs to be made on all the copies, which is almost impossible to do simultaneously.

The rest of this paper is organised as follows. Section 2 reviews the background of blockchain. Section 3 describes the blockchain architecture. Section 4 details the impact of blockchain in accounting. Section 5 discusses the challenges blockchain is posing. Future directions are given in Section 6. Finally, Section 7 concludes the paper. 


\section{Background}

Blockchain technology's existence goes back to 2008 and has recently taken the industries by storm. Many industries have started to implement blockchain solutions in some of their business cases. The first blockchain technology was founded shortly after the US financial collapse in 2008. Nakamoto (2008) introduced Bitcoin. Bitcoin is a cryptocurrency that uses traditional cryptography to manage the production and transfer of money. It uses a peer-to-peer (P2P) Bitcoin network to send decentralised digital currency from one user to another.

Blockchain can be visualised as an open book that contains a record of all the exchanges and interactions between users that took place through it. It consists of a P2P network and a decentralised distributed database (Khan, 2017). There is no central authority to supervise the blockchain ledger. In other words, blockchain is simply a chain of blocks, each block is made of a header and body. It exists on a P2P network where every node stores a local copy of the public ledger. Each record in the ledger is called a block and includes details such as the transaction timestamp, hash pointer to its previous block, as shown in Figure 1. The hash pointer makes it impractical for anyone to change information about the transactions retrospectively. Also, the technology is safe by design, as the database system of multiple nodes records the same transaction. In this manner, the information can never be altered or erased. Blockchain systems are categorised into three types, private, public, and consortium blockchain (Zheng et al., 2018). The comparisons between three kinds of blockchain systems based on different features are listed in Table 1.

Table 1 Comparison between private, public, and consortium blockchain

\begin{tabular}{lccc}
\hline Feature & Private blockchain & Public blockchain & Consortium blockchain \\
\hline Access & Private & Public & Private/public \\
Consensus & Organisation-based & Public & Selected nodes \\
Efficiency & High & Low & High \\
Centralisation & Yes & No & Partial \\
Consensus process & Permission-based & Permission less & Permission-based \\
Immutability & Not completely & Completely & Not completely \\
& tamper-proof & tamper-proof & tamper-proof \\
\hline
\end{tabular}

Bitcoin was the first real-life blockchain application (Peck, 2017). Bitcoin can solve the problem of 'double spending' in the traditional payment method without a trusted third party's aid. Usually, when people make payments, a trusted third party is involved in verifying the transaction's validity before putting them into action. For example, to complete an electronic transaction, there must be a way to validate the funds transmitted have not been already spent. Conventionally, this problem is solved by using trusted third parties like Propay, Paypal, and Venmo, which verifies and validates each transaction and maintains a public ledger of the user's transaction to prevent double-spending (Pacy, 2014).

However, it is the third party that is mostly in doubt as to whether it could be exploited to commit fraud (The Economist, 2015). The problem comes from formal centralisation, in which everything depends on a single organisation, causing the trust to be insufficient. Bitcoin offers a different solution to this problem, using cryptographic 
proof instead of trust. To create and maintain the public ledger, known as the blockchain, bitcoin relies on a P2P network and decentralised distributed database. This is in contrast to a centralised database that is in use presently. Each transaction contains two public keys- one each from the sender and receiver-proving that the funds were transferred. Both parties have to sign the transaction with their private key, and the transaction is recorded in the block. The block is then verified by the network before adding to the blockchain (Pacy, 2014).

Several business houses, including brick and mortar stores, recently began accepting Bitcoin as payment. Some of the well-known Bitcoins acceptors are Microsoft, Subway, Overstock.com, Expedia, and Newegg. People used Bitcoins to buy drinks at pubs, cars from the dealerships, even as real estate payments (Beigel, 2020). Since 2009, many Bitcoin variants have been introduced, including Ethereum, Bitcoin Cash, Ripple, and many others. As a currency, Ethereum features smart contracts, also known as Ether. It enables users to publish censor-secure software programs that can be operated infinitely in the absence of its creator. Together with the smart contract, Ethereum proved to the world that blockchain technology could be a viable option to many real-world situations other than being an alternative to the conventional financial system. Not only has this technology been implemented in sectors including consumer products, finance, manufacturing, media, telecommunications, but also in healthcare, travel, and government since 2014. IBM has teamed up with the Linux Foundation on the Hyperledger project, which is developing an open-source, standardised, and production-grade digital ledger.

\section{Blockchain architecture}

A blockchain is composed of a network of nodes and databases. Its database is distributed and follows an append-only notion. In the high-level blockchain, architecture is divided into six different layers, including, data layer, network layer, consensus layer, contract layer, service layer, and application layer as illustrated in Figure 2 (Lu, 2018).

Data and network layers are bottom-most, and these lower layers are responsible for generating, validating, and saving data and information. The consensus layer includes consensus mechanisms such as proof of work (PoW), proof of stake (PoS), and delegated proof of stake (DPoS), and practical Byzantine fault tolerance (PBFT), which helps the interconnected nodes to reach consensus. This layer also functions as a bridge between the lower and upper layers. Similarly, the contract layer includes smart contracts (a set of a predefined set of rules), control logic, consensus protocols, and incentive mechanisms. The top-most layer is the application layer, which mainly covers applications and services (Khan et al., 2020).

\subsection{Data layer}

The data layer includes various elements, for instance, data block, chain structure, timestamp, digital signature, hash function, and Merkle tree.

- Data block: It is used for the recording of interactive data and information. In the blockchain ecosystem, the raw data at the bottom level has to be further processed 
for storage in the block. A block contains a block header as well as the block data. The block header contains metadata for this block as shown in Figure 3.

Figure 2 Blockchain architecture (see online version for colours)

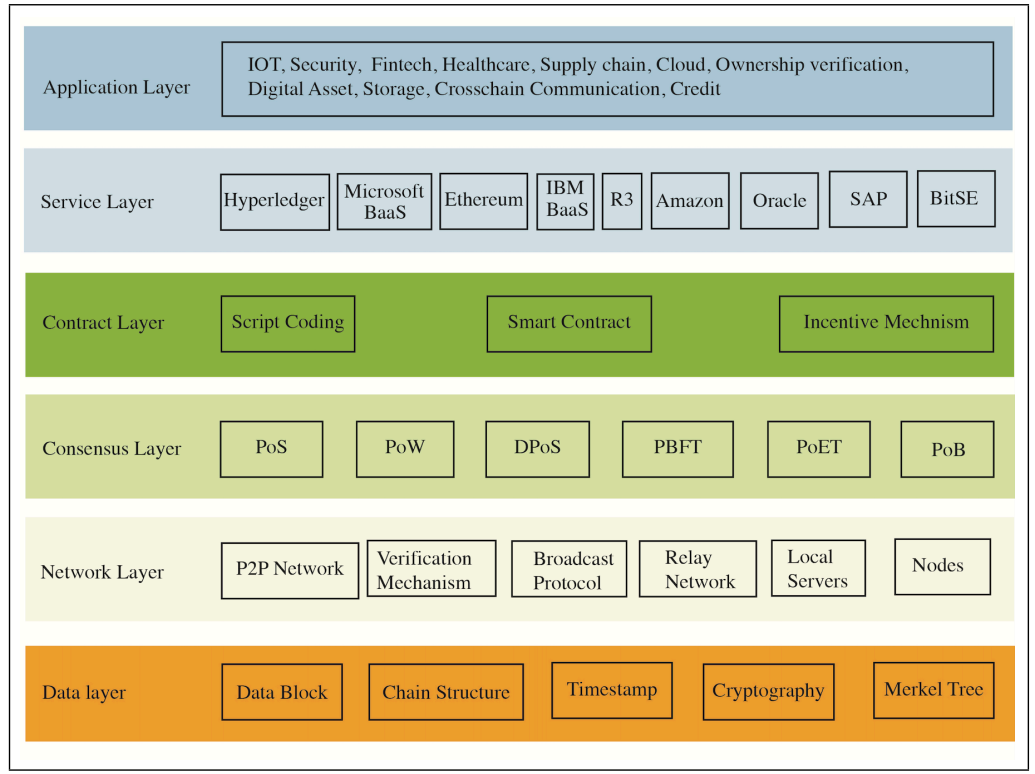

- Chain structure: The name is derived from the structure of each record, called blocks, which is linked to a single list, known as a chain (Christensson, 2018). A block contains the header of the block and the body of the block. The header is made up of several other components, while the body of the block has a transaction counter that records every transaction. As blocks are added to the list it forms a sequential chain-like structure as depicted in Figure 4.

1 Block version: Specifies which set of rules to follow for block validation.

2 Merkle tree root hash: The hash value of every block transaction. is also known as the Tx_Root. It includes the hash value of all validated transactions of the block. As illustrated from the example in Figure 4, all the transactions are hashed into a hash value; then, they combine pair-by-pair and are inputted to another hash function. This work is repeated until there is only one single entity, which stands for the Merkle root.

3 Timestamp: Time in seconds between the current time and the Unix Epoch, that started on 1 January 1970. It refers to the time when the block was found.

4 nBits: The target threshold for a valid block hash.

5 Nonce: A field of 4 bytes usually starting at 0 and increasing with any calculation of the hash. This field is used in PoW, which demonstrates the efforts made by a node to obtain the right to append its block to the chain. 
6 Parent block hash: A 256-bit hash, pointing to the previous block.

A mathematical algorithm is used to map the data in the block to a fixed-length binary digit string known as the cryptographic hash. For integrity and event order, blockchain uses the SHA-2 hash function. SHA-256 and SHA-512, are the most commonly used versions for hashing. SHA-256 algorithm produces a 256-bit cryptographic hash.

Figure 3 Block header (see online version for colours)

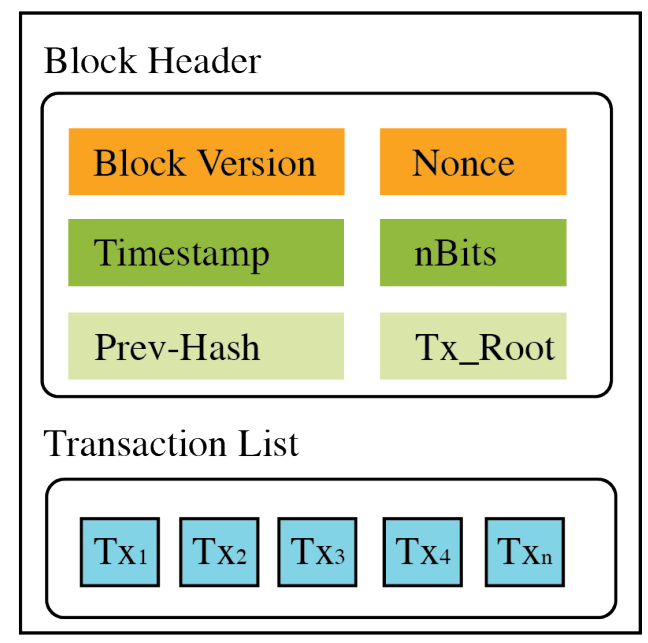

Figure 4 An example of a sequential chain of blocks in the blockchain (see online version for colours)

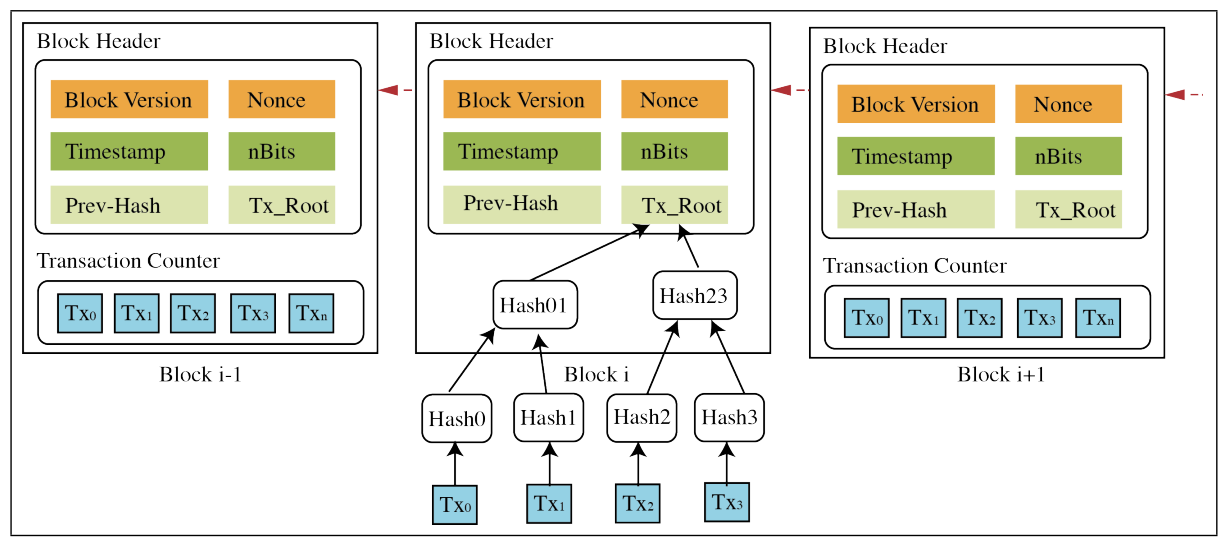

The first block of the blockchain is called a genesis block. The blockchain is envisioned as a vertical stack, and the blocks are stacked on top of one another, with the genesis block being the base of the stack. The block body is composed of a transaction counter and transactions. The maximum number of transactions that a block can contain depends on the block size and the size of each transaction (Zheng et al., 2017). Blockchain uses asymmetric encryption and hashing algorithms to verify the untrusted network and ensure security in such networks (Lu, 2018). 


\subsection{Network layer}

Blockchain nodes are interconnected by a P2P network. P2P network is an overlay network built up over the internet. The P2P blockchain can be modelled as structured, unstructured, or hybrid depending on the type of blockchain and its parameter. However, a synchronisation protocol is needed to publish a newly created block in the chain, but routing protocol may or may not be needed. For example, Bitcoin does not require a routing protocol, as a peer can receive information at most in one hop in the network (Raikwar et al., 2019). A broadcast protocol is needed to broadcast two types of information: information about interactions and information about blocks. Unlike bitcoin, which uses unstructured P2P networks, fintech companies should adopt the use of structured P2P networks. Using a structured network will enable companies to locate resources easily as each node has an identifier with the routing table.

Figure 5 Consensus algorithms (see online version for colours)

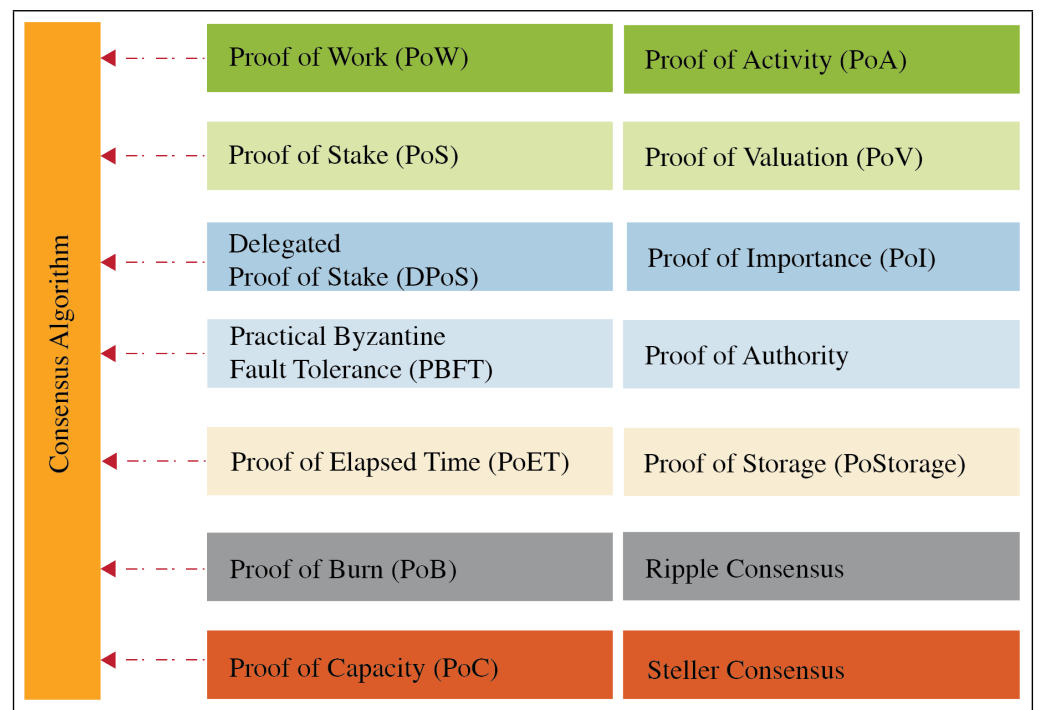

\subsection{Consensus layer}

Adversaries can act maliciously in many ways in an open, trustless, and distributed network. Bitcoin uses the PoW protocol to reach consensus, which requires a node to try and solve a hard-computational problem to validate a batch of transactions and add them to the blockchain as a new block. Many variants of proof-based consensus algorithms have been proposed, which are based on PoW, PoS, and their hybrid form. The other variants that are made independently from PoW and PoS are shown in Figure 5. The basic concept of the proof-based consensus algorithm is that the node that performs sufficient proof will get the right to add a new block to the chain and receive the reward among many nodes that join the network. We have opted to define the PoW consensus algorithm in this paper as it is the most commonly used at present. 


\subsubsection{PoW-based consensus algorithm}

Each node needs to show a certain amount of work done, also known PoW to add new blocks to the blockchain. In the blockchain network, if every node tries to broadcast their blocks containing the verified transactions, a possible ambiguity is created. For instance, if a transaction is verified by several nodes, placed in their blocks, and then transmitted to other nodes, this transaction may be duplicated into different blocks, then the ledger becomes meaningless. The participating nodes would have to put their verified transactions, as well as other information like Prev_Hash and Timestamp, into a block before they start solving the puzzle. The puzzle (secret value) is a nonce field that is put back into the block after the node successfully finds it. After this, all the information inside the block header will be combined and inputted into an SHA256 hash function. The secret value is accepted if the output is below the given threshold $\mathrm{T}$, designated by the difficulty; otherwise, the nodes will still need to devise the secret value (Nguyen and Kim, 2018). For Bitcoin, the node must consider a hash value that is less than a certain number, often referred to as the network's fixed level of difficulty. The degree of complexity is automatically determined by the Bitcoin algorithm, which means that a new block is generated every 10 minutes. The process of solving the PoW puzzle to find a winning hash value is called mining. The first node to find a winning hash adds the block to the blockchain and claims the mining reward as well (Baliga, 2017). When a node solves the problem, it broadcasts its proposed block with this value to other nodes to notify them that the problem has been solved. Right after that, all the miners receiving this message, who still have not resolved the problem, will stop solving. Instead, they start checking the validity of the broadcasted block. The validity is checked by comparing the hash value of the previous block. This ensures integrity in the chain. Once validated by every node in the network, the proposed block is finally appended to the chain. This process repeats over and over again. Given that this process is distributed and concurrent, several nodes can find a winning hash at the same time. The winning nodes typically add their block and broadcast it to the blockchain via a P2P network. In this case, there is a temporary fork, as shown in Figure 6, the chain is branched out, and new blocks are added to different branches. The miners will continue mining new blocks to the forked branch, but the protocol will ensure that the longest branch with PoW will be included in the chain while shorter ones are discarded subsequently. This ensures coherence between all the nodes regarding the state of chain (Baliga, 2017).

\subsection{Contract layer}

The contract layer contains multiple script codes and algorithms that are required for blockchain operations and smart contracts. The contract layer is a rule-based protocol that has been developed to allow flexible programming and operational data functions (Puthal et al., 2018). 
Figure 6 Forking problem

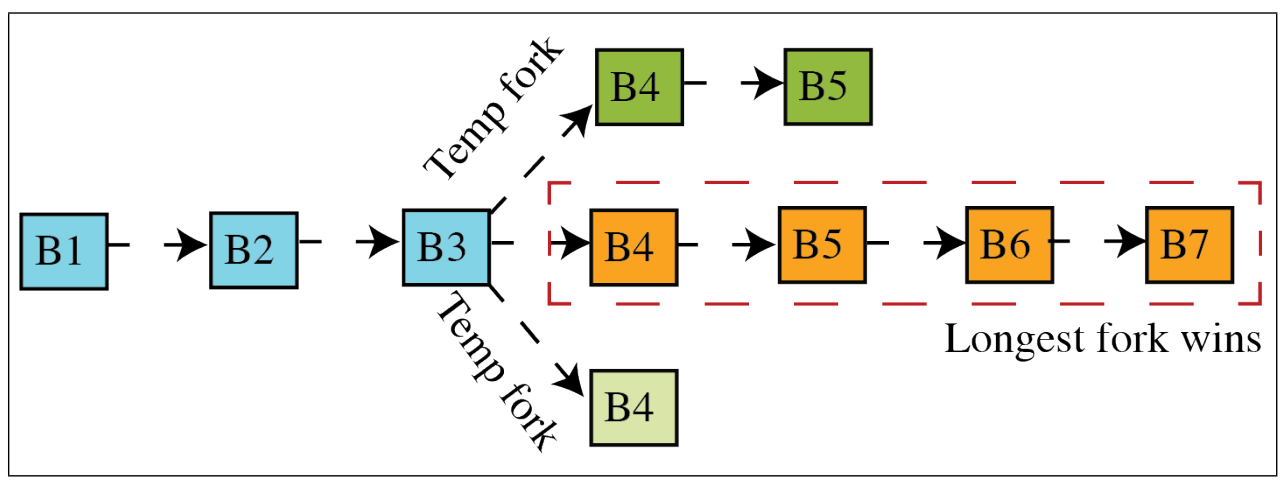

\subsubsection{Smart contracts}

In the blockchain context, smart contracts are much more comprehensive than traditional transactions to buy and sell. Apart from the purchase and sale, they may have extensive sets of instructions integrated into them. A contract is a more formal way of using Bitcoin for concluding agreements with individuals through blockchain. A traditional contract is a deal between two or more parties to do something or not in exchange for something. Each party must have confidence in each-other to meet their side of the obligation for the exchange. Smart contracts have the same sort of agreement to act or not to act, but they eliminate the need for one kind of trust between the parties in an agreement. This is because the smart contracts are both defined and executed by the code automatically at no discretion. Three elements of smart contracts that make them distinct are autonomy, self-sufficiency, and decentralisation (Swan, 2015).

The decentralised blockchain ensures that honest parties obtain adequate compensation in case of contractual breaches or abortions. There is, however, a lack of transactional privacy in existing systems. Every transaction in the blockchain is exposed, which includes cash flow between aliases and amounts transacted (Kosba et al., 2016). A solution to transactional privacy for accounting firms is to adopt a private smart contract that does not store transactional details in plain text on the blockchain rather applies a cryptographic solution to parties in a contract.

\subsection{Service layer}

The service layer is a mixture of computational resources and lower-level data that provides services for top-level specific application services (Gupta et al., 2018). Companies build a service platform independently. Some of the examples of a blockchain platform developed by companies today include IBM Azure BaaS, Ethereum, and Hyperledger (Castillo and Schifrin, 2020). 


\subsection{Application layer}

The application deals with how blockchain can be used across different platforms. Some of the blockchain's use cases include accounting, supply-chain, medical care, insurance, government, cloud services, etc.

\section{The impact of accounting}

Blockchain technology has the potential to impact a wide range of industries. It is expected that blockchain will improve collaboration among businesses, the transparency of business processes, and, ultimately, the productivity and sustainability of the economy. Blockchain works even better with applications which involve the transfer of value and asset between parties. At present, such transfers in businesses are cumbersome, costly, and take one or more centralised organisations. For example, securities settlement generally includes multi-day clearing and settlement processes between several financial intermediaries. Some financial services specialists believe that the financial services industry is on the brink of disruption: advances in emerging technology like blockchain are projected to transform the industry and its workforce by automating many of the activities currently performed by humans (Bible et al., 2017).

Blockchain can be viewed as accounting technology. It is concerned with the transfer of ownership of assets and maintaining a ledger of accurate financial information. The accounting profession is largely concerned with the measurement, communication, and analysis of financial information. Most professionals work to ascertain or measure property rights and obligations or to plan the best allocation of financial resources. For accountants, the use of blockchain brings transparency about ownership of property and bonds. By knowing obligations and transparency in transactions, an employee can function better and become more productive. This will dramatically improve work efficiency in turn. Blockchain has the potential to enhance the accounting profession by reducing the costs of maintaining and reconciling ledgers and providing absolute certainty over the ownership and history of assets (ICAEW, 2017).

To understand how blockchain can be beneficial to traditional bookkeeping systems, we need to understand how transactions are entered and kept by business organisations in the past and present. Three important methods of bookkeeping are discussed below to showcase how accounting information is stored, verified, reconciled, and audited.

$1 \quad$ Single entry system: In single-entry bookkeeping each business transaction is recorded in only one account. Although this mechanism is fast and simple, it was fraught with errors, be it accidental or fraudulent. Errors and frauds are common in this method of bookkeeping because of the absence of cross verification (Grigg, 2005).

2 Double entry system: To address the issue of inaccuracies and errors about single-entry bookkeeping systems, accounting today is based on double-entry systems. At least two accounting entries are required for recording and documenting each financial transaction in the double-entry accounting system. Such entries may be in the account of assets, liabilities, equity, expenditures, or profits. A debit amount for one or more accounts and a credit equal for one or more accounts results in total credits equal to all accounts in the general ledger. In 
case of an error-free recording of accounts, the total balance of all accounts with debit balances equals the aggregate balance of all accounts with a credit balance. Debit and credit accounts accounting entries normally have the same date and identification code in the two accounts so that every debit and credit can be traced to the journal and the transaction source and thereby establish an audit trail in the event of an error. These accounting entries are then entered in the journal. The basic accounting equation of assets equal liabilities plus capital would hold regardless of which accounts and how many are affected by a given transaction. Public auditors often check and validate the financial details of the company to gain the trust of outsiders. This is a costly and rigorous process that will not only bind the human resources (accountants, bookkeepers, and financial officers) of the company but also take away their precious time to help auditors (Inghirami, 2020). Grigg (2005), in his paper on 'Triple entry accounting' argues that "it adds an important property to the accounting system; that of a clear strategy to identify errors and to remove them. Even better, it has a side effect of clearly firewalling errors as either accident or fraud." This method may minimise human documentation errors and inconsistencies but may not provide full assurance of the financial statement of the company.

3 Triple entry system: To enhance the reliability of corporate financial statements, this bookkeeping system has been suggested as an independent and safe paradigm. This method requires a neutral third party to oversee and record each accounting transaction meaning three entries were created, two entries were recorded by transacting parties, and third entry was recorded by an intermediary. However, there are concerns regarding the use of third parties as they are vulnerable to cyberattacks. The next step in the accounting process may be blockchain technology: rather than holding independent documents based on transaction receipts, businesses may write their transactions directly into a joint ledger and establish a continuously interlocking accounting system. Because all the entries are circulated and encrypted, it is almost impossible to forge or delete them or cover up activity. With blockchain's advent, processes can become automated, cheap, and even more cost-effective. A decentralised ledger supersedes the need for third parties who keep receipts centrally. Also, smart contract technology could enable rapid verification of transaction records per accounting standards or business rules. Transparency of blockchain gives visibility for all authorised user's transactions, which can minimise auditor's work with sampling and validating transactions. This allows auditors to focus more time on inspections and anomalies. Meanwhile, CPAs have the potential to leverage blockchain technologies to apply their assurance offerings to the fields of cybersecurity and sustainability (Inghirami, 2020).

Blockchain can transform the way financial records are created, maintained, and updated. Instead of one owner, blockchain records are distributed to all users. This can be viewed as a 'universal entry bookkeeping' where each participant has access to every entry. As discussed earlier, it uses a system of consensus and verification to ensure that a permanent record is transmitted to all users. Unlike traditional ledgers, which are database focused and run by a single centralised party, it is decentralised and offers a different perspective to data and transaction. For instance, decentralised consensus could eventually improve the employee's engagement in accounting issues 
and verification of company transactions, shifting to more diversified controls by blockchain-led transparency (Rückeshäuser, 2017).

Another critical issue that the accounting industry is currently facing is financial transparency. There is very little or no financial transparency between the transacting parties at the moment. Also, the fact that current systems lack real-time data and information. This issue could be addressed through the use of blockchain. The most important features of blockchain are propagation, permanence, and programmability. Propagation is the access ability to an identical full copy of the transaction records to all participants. Permanence signifies that past transactions cannot be edited without the approval of the majority, which makes blockchain records permanent. Programmability allows program code to be stored with the ledger entries to create automatic journal entries, also known as smart contracts. Propagation and permanence are essential to the blockchain. Any application based on blockchain has these key characteristics.

Blockchain's distributed ledger for transactions can replace central authorities such as banks, clearinghouses, and lawyers. Also, the fact that parties can interact directly with one ledger, which means there is never a need for reconciliation; this in turn, could save money and time for a business. Moreover, tax authorities and regulators having access to blockchain could observe and monitor transactions in real-time, which will not only be cost-effective, but it will also increase the efficiency of regulatory and compliance activities. The permanent record of a blockchain reduces the risk of financial crime, which makes records trustworthy. The technology supports a fast clearing and settlement process. Once a transaction is confirmed and submitted to the ledger, it also settled in the wallet. This fast settlement leads to cost reduction and lowers the risk of fraud. Blockchain protocol facilitates verification before each block is written to the ledger. Furthermore, it supports document retention. Any authorised participant can access and verify ownership records, including transaction information and procedures embedded in smart contracts. Blockchain also contains detailed information such as precise histories of asset movements and time stamping that can hugely benefit the auditing process. Another benefit is the operational improvements. Blockchain adoption can eliminate some middle and back-office processes, which include not only reconciliation, but it also aids in error correction and improve business.

To be effective, it is important to note that any accounting company which wishes to use technology blockchain needs to choose a private blockchain as its platform. Using private blockchain has various advantages over public blockchains. Any interested party may enter or leave the public blockchain network at any point that is undesirable in an accounting context. Another thing that accounting firms want to avoid is the transparency of financial and transactional records to the public (Appelbaum and Smith, 2018). The information is available for anyone interested, be it financial or transactional. It raises questions about the protection of privacy. To prevent this, accounting firms should consider using a private blockchain solution with shared access to agreed parties. The genius of a private blockchain is that it not only safeguards the confidentiality of the transaction among approved stakeholders but also limits who can join the network. Private blockchain participants are granted specific network rights and restrictions so that someone has either full or limited access at the network's discretion. This feature of different levels of access rights provided to different users in private blockchain can be viewed as role-based access control (RBAC).

Thus, depending on the functionality of the organiser, the information types stored and circulated over the blockchain, and the different levels of access that 
different stakeholders need, private blockchains appear to be the most logical and business-friendly option for accounting firms (Intuit, 2020).

\subsection{How big four accounting firms use blockchain?}

At this point, at least all big four accounting firms (Deloitte, Ernst \& Young, KPMG, and $\mathrm{PwC}$ ) have shown some interest in blockchain, although they tend to differ in interest and approach. In this paper, we looked into the details of the blockchain adoption of each firm.

1 Deloitte: The earliest one to dive into the crypto space was among big four is Deloitte. Back in May 2016, they announced their first blockchain lab in Dublin, Ireland. It collaborated with the Bank of Ireland to conduct joint proof-of-concept based blockchain trial. Currently, three of the four largest banks in Ireland are reported to be using blockchain Deloitte's to verify the credentials of their employees (O’Neal, 2019). They also launched Rubix, an enterprise blockchain in Toronto in 2014. Rubix offers advisory services and creates decentralised applications based on a blockchain platform for clients from different industries. It was initially catered to four specific types of applications: reconciliation, audits, land registry, and loyalty points (Vetter, 2018)

2 Ernst \& Young: EY in Switzerland was the first of 'big four' accounting firm to accept bitcoin for both auditing and advisory services. The firm introduced the EY Blockchain Analyzer back in 2018, which has been developed utilising experience gained from working with global clients in the USA, Europe, and Japan. According to EY's (2018) press release "EY Blockchain Analyzer is a suite of blockchain audit technologies that improve the ability to perform an in-depth review of cryptocurrency business transactions."

EY has been continuously developing blockchain-based solutions. Some of the other blockchain applications created by EY include nightfall, dye pack, and smart contract and token testing.

3 KPMG: Not only has PMG increased its presence in the blockchain space, but since 2017 it has also been part of the Wall Street Blockchain Alliance (WSBA) (Das, 2017).

In 2016, KPMG launched a digital ledger services program to assist financial services companies to investigate blockchain. According to KPMG itself, the "Digital Ledger Services enable financial institutions to realise the potential of blockchain capabilities in the cloud, including faster and more secure transactions, streamlined and automated back-office operations, and reduced costs." KPMG also partnered with Microsoft Corp. to launch the "blockchain Nodes" initiative designed to create and identify new applications and use cases for blockchain technology. However, the latest survey by KPMG on blockchain showed that many tax and financial managers do not plan on blockchain technology adoption (Perez Yessi, 2019).

$4 \quad P w C$ : PwC was the world's second largest accounting firms to accept bitcoin payments for its services in Hong Kong (Das, 2017). The firm supported various 
blockchain projects and introduced a blockchain audit service that will encourage people to use the technology. According to the firm, the audit service needs an independent validation. The decentralised digital ledger can also be used for online identity verification, supply chain management, and auditing financial transactions, which is part of an auditor role. PwC also logs transactions on the blockchain and developed testing criteria and controls. Thus, users within a company can view, test, and monitor transactions on the blockchain in real-time.

PwC views blockchain as the software to structurally modify shared traditional practice between clients, competitors, and suppliers for the next-generation business process but also believes that development in blockchain solutions is not taking a rapid pace because of its regulatory uncertainty (PwC, 2018).

Table 2 Comparison of BaaS platform

\begin{tabular}{|c|c|c|c|c|c|}
\hline & Microsoft & Amazon & $I B M$ & Oracle & $S A P$ \\
\hline $\begin{array}{l}\text { Major } \\
\text { partner }\end{array}$ & $\begin{array}{l}\text { Cords, } \\
\text { Blockapps, } \\
\text { GoChain, } \\
\text { Consensys }\end{array}$ & $\begin{array}{l}\text { Cisco, Intel, } \\
\text { Corda, R3, } \\
\text { Blockapps }\end{array}$ & $\begin{array}{c}\text { SecureKey } \\
\text { Technologies, } \\
\text { Canadian banks }\end{array}$ & $\begin{array}{c}\text { ICS FS, } \\
\text { Vottum, myEtf, } \\
\text { HACERA, } \\
\text { Deloitte, } \\
\text { Infosys }\end{array}$ & $\begin{array}{c}\text { Intel, UPS, } \\
\text { HPE and } \\
\text { Airbus }\end{array}$ \\
\hline Major user & Xbox, 3M & $\begin{array}{l}\text { T-Mobile and } \\
\text { Guide Wire }\end{array}$ & $\begin{array}{l}\text { Intelipost, } \\
\text { Nigeria } \\
\text { Customs }\end{array}$ & $\begin{array}{c}\text { Circulor, } \\
\text { SERES, and } \\
\text { CDEL } \\
\text { HealthSync }\end{array}$ & - \\
\hline $\begin{array}{l}\text { Authentication } \\
\text { and } \\
\text { authorisation }\end{array}$ & $\begin{array}{c}\text { Active } \\
\text { Directory }\end{array}$ & $\begin{array}{l}\text { Identity and } \\
\text { Access } \\
\text { Management }\end{array}$ & $\begin{array}{l}\text { IBM Secure } \\
\text { Service } \\
\text { Container }\end{array}$ & $\begin{array}{c}\text { Identity } \\
\text { Federation, OCI } \\
\text { Identity Cloud } \\
\text { Service }\end{array}$ & Service Key \\
\hline $\begin{array}{l}\text { Blockchain } \\
\text { type }\end{array}$ & Permissioned & Permissioned & Permissioned & $\begin{array}{l}\text { Permissioned } \\
\text { Consortium }\end{array}$ & Permissionless \\
\hline $\begin{array}{l}\text { Development } \\
\text { facility }\end{array}$ & $\begin{array}{l}\text { High (with MS } \\
\text { development } \\
\text { kit) }\end{array}$ & $\begin{array}{c}\text { Medium } \\
\text { (limited only } \\
\text { with AWS kit) }\end{array}$ & $\begin{array}{c}\text { IBM mix } \\
\text { development } \\
\text { platform }\end{array}$ & $\begin{array}{c}\text { Hyperledger } \\
\text { Fabric SDK, } \\
\text { Oracle Visual } \\
\text { Builder Studio } \\
\text { CS }\end{array}$ & Not released \\
\hline Scalability & $\begin{array}{l}\text { High (with all } \\
\text { MS product) }\end{array}$ & $\begin{array}{l}\text { Provide API for } \\
\text { quick node } \\
\text { creation }\end{array}$ & $\begin{array}{l}\text { IBM Smart } \\
\text { Cloud only }\end{array}$ & $\begin{array}{c}\text { Dynamic } \\
\text { scale-out and } \\
\text { scale-up in the } \\
\text { cloud and } \\
\text { on-premise }\end{array}$ & - \\
\hline
\end{tabular}

\subsection{Blockchain adoption: blockchain as a service}

"Blockchain as a service (BaaS) is a type of blockchain service offering that allows business customers to use cloud-based solutions to develop, host, and adopt their blockchain applications, smart contracts, and other relevant functions on the blockchain while the cloud-based IT partner or service provider manages all the required tasks and activities to keep the infrastructure up and running." (Leewayhertz, 2020) 
Blockchain is becoming more and more accessible to businesses (small to large scale) because BaaS offerings like IBM's BaaS (Hyperledger) and Ethereum Enterprise Alliance (EEA) significantly reduce the cost and overheads of adoption. Essentially, BaaS involves a service provider that offers and manages various DLT infrastructure components.

IBM's BaaS is based on the open-source Hyperledger Fabric, version 1.0 from The Linux Foundation. It is a public cloud service that customers can use to build secure blockchain networks. EEA aims to build on and adapt Etherum (which includes Ether, a token/bearer-asset) to address business needs such as permission management, whereas Hyperledger focuses on permissioned chains without a crypto-currency basis (tokens) (Singh and Michels, 2018). The precise nature of a BaaS deployment depends on embracing the company's end requirement, the service provider they choose, and application specifics. Today, many established tech giants such as Microsoft, IBM, Amazon, Oracle, and SAP offer BaaS. In this paper, we have compiled a list of some of the popular BaaS platforms based on the features such as major partners, major users, authentication and authorisation, blockchain type, development facility, and scalability as illustrated in Table 2 .

\section{Challenges}

Blockchain technology aligns seamlessly with the accounting profession. It gives users the ability to create value and authenticate information quickly and precisely without the threat of being corrupted. Despite having many benefits and use cases, there are, however, some challenges and limitations. Some aspects of the technology may require further development to reach its full potential. In this paper, the challenges, both technical and business, are divided into five main categories as follows:

\subsection{Scalability}

Scalability is a characteristic of a system, model, or function that describes its capability to cope and perform well under an increased or expanding workload or scope. A system that scales well will be able to maintain its performance and efficiency even if it is tested by large and concurrent operational demands (Hayes, 2020). In other words, it is defined as the capacity for a system to continue to function to its extent when the system considerably changes in volume or size-usually, to a larger volume or size. Scalability issues arise due to existing consensus methods and the size of the block where each node in the network sequentially authenticates the transaction before publishing it to the blockchain. This problem is compounded with the increasing number of transactions requiring more nodes to support the network, while at the same time increasing the number of steps for the transaction to travel and reaching full consensus with each node (Chauhan et al., 2018). Scalability has three major component latency, size and storage, and throughput.

\subsubsection{Latency}

Latency in the blockchain domain is defined as the time required for a transaction to commit. Currently, blockchain has a significant latency as compared to other e-payments 
giants such as VISA and PayPal. At the moment, Bitcoin blockchain guarantees mere 4.6 transactions per second, whereas VISA and Paypal process around 1,736 and 193 transactions per second (tps) on an average, respectively (Li, 2019).

\subsubsection{Size and storage}

The challenge is also that transactions can produce large volumes of data, but the blockchain is not sufficiently scalable to store big data. One of the characteristics of blockchain is decentralisation, and this means that every single node on the network processes every transaction and maintains a copy of the entire state. The number of transactions it can process has a limit, and as more nodes are added to its network, the blockchain becomes weaker. Therefore, as the size of the blockchain grows, the requirements for storage increase.

\subsubsection{Throughput}

As of today, the theoretical peak throughput of Bitcoin is about seven transactions per second (Blocksplain, 2018). Blockchain consensus technologies such as PoW and Byzantine fault tolerance (BFT) sit at the two opposite ends of the scalability spectrum. PoW-based blockchain offers good node scalability with poor performance, whereas BFT-based blockchain offers good performance for small numbers of replicas, with not-well explored and intuitively very limited scalability. Performance depends greatly on the blockchain's deployment. For example, a hybrid blockchain can be designed to perform much better than a public blockchain. To solve the problems of performance, private blockchains are a viable alternative to the accounting industry (Vukolić, 2015).

\subsection{Privacy leakage}

Most blockchains are massively distributed and publicly accessible databases. It is almost impossible to keep secrecy in public blockchains because those blockchains are meant to be public. Attackers can effectively use publicly available information to establish the link between the sender and the receiver. In this sense, blockchain adopters like Bitcoin are less private than traditional banking, where federal regulations mandate basic privacy protections (Henry et al., 2018). Research is still ongoing on cryptographic solutions that can prevent the leak of private and personally identifiable material about transacting parties.

\subsection{Regulatory issue}

Several states, the federal government, and international agencies have shown interest in regulating blockchain activities with a focus on virtual currencies. The widespread application and relative newness of blockchain technology make it impossible to conclude which agencies are likely to investigate specific blockchain activities. Mostly because the scope of regulatory authority in this field is yet to be defined, and blockchain activities may involve multiple agencies' jurisdiction. It is likely, a regulator will investigate any blockchain activity that falls within its traditional jurisdiction (Michael et al., 2018). PwC in its 2018 report suggests that regulatory uncertainty is 
the major hurdle of blockchain adoption (Stanley, 2018). Blockchain's potential is much bigger than cryptocurrency; if regulatory uncertainty alleviates, the blockchain's value can easily increase by hundred-fold to $\$ 3$ to $\$ 4$ trillion by 2030 (Taskinsoy, 2019).

\subsection{Standardisation}

Standardisation provides a basis for technology investment and economic activity. Thus, these standardisation efforts should be based on best practice and be readily applicable. Blockchain is novel and best practice continues to evolve rapidly. However, a major standardisation initiative has been initiated on blockchain technologies through a technical committee of the International Organization for Standardization (ISO), ISO/TC 307. Other organisations like the Institute of Electrical and Electronics Engineers (IEEE) and the International Telecommunication Union (ITU) have also established standardisation efforts on blockchains. The National Institute of Standards and Technology (NIST) has recently announced that it will collaborate with ISO to define blockchain standards. The United Nations Economic Commission for Europe (UNECE) is also studying the use of blockchain for trade-related use cases (Gramoli and Staples, 2018). Standardising the blockchain's definition, its components such as clients and servers, consensus, pseudonymity, validation, transaction, smart contract, etc. will undoubtedly help the business organisation adopt blockchain more efficiently and seamlessly.

\subsection{Financial and human capacity}

Small accounting and financial organisations usually are resource-constrained. These companies, in general, do not have sufficient financial capacity to implement new technology and must, therefore, rely on their legacy accounting systems to operate daily. In contrast to Delliot and PwC, the majority of companies do not have the financial capacity to examine emerging trends. Another concerning challenge is the human capacity within the organisation. Regardless of the benefits, workers are so embedded in the legacy business models; it is quite daunting for their employer to alter their mindset in embracing new technology. There is also the fear of smaller companies not using technology at the same pace as bigger companies. This, in-turn, means larger organisations might have to wait for smaller parties to adopt blockchain. Unless the same blockchain platform is used by all trading organisations, we cannot fully evaluate blockchain's potential.

\section{Future directions}

\subsection{Blockchain with AI}

Artificial intelligence (AI) is expected to change the workplace and life in general. An AI-based business application will be groundbreaking to many organisations and allow them to transform their organisation into innovative, efficient, and sustainable organisations. Leveraging the AI along with blockchain will help improve productivity and build a competitive value chain. To embrace the new technologies without business 
interruptions will be challenging for the companies. However, to make the transition smooth, companies need to deploy blockchain experts in core lines of businesses such as audit, tax, industries, etc. Blockchain, on the one hand, suffers from weaknesses such as security, scalability, and efficiency, while AI, on the other hand, has its fair share of issues with trustworthiness, explainability, and privacy. The integration of these two systems appears to be unavoidable; they could balance each other to revolutionise the next digital global age. Trustlessness, privacy, and explainability for AI are inevitably brought by blockchain. AI can, in turn, help build a blockchain machine learning system to improve security, scalability, governance, and personalisation (Dinh and Thai, 2018).

Kim et al. (2019) proposed a privacy-preserving distributed machine learning (DML) model for a permissioned blockchain to resolve the privacy, security, and performance issues with differential privacy (DP). The model is based on the simulate-order-execute architecture of Hyperledger Fabric. Their method is based on two primitives, a differentially private stochastic gradient descent method and an error-based aggregation rule. The experimental results indicated the proposed model provides stronger resilience against adversarial attacks and has high usability because it has low complexity and low transactional latency.

Salimitari et al. (2019) introduced AI-enabled blockchain (AIBC) with a two-step consensus protocol that uses an outlier detection algorithm for consensus in an IoT network implemented on Hyperledger Fabric platform. According to the researchers, the outlier detection algorithm acts as the first step consensus and verifies the compatibility of new data and discards the suspicious ones to increase the fault tolerance of the network for the second step consensus (PBFT). They measured the latency, accuracy, and performance of their method. Experimental results suggested a significant increment in the fault tolerance of hyperledger fabric by their proposed detector.

\subsection{Improving scalability}

Scalability is one of the main constraints of a blockchain. Blockchain systems like Bitcoin and Ethereum offer strong guarantees but have a limited capability of processing transactions with the same efficiency as other distributed systems. Throughput is also limited by the size of blocks and the rate at which blocks are produced. Blockchain transactions also suffer from high latency. A user has to wait for a confirmation block before the transaction is posted to the chain. Alternative consensus mechanisms can address the issue of latency but cannot improve throughput without making adjustments in blockchain design itself. Several other techniques have been proposed to improve blockchain throughput, including two broad classes such as block graph systems (Sompolinsky and Zohar, 2018) and sharding (Zamani et al., 2018). Graph-based systems have explored generalising the structure of the relationships between blocks from a strict chain to a graph. Other systems improve throughput by relaxing the constraint that every transaction is propagated to and processed by the entire network, known as sharding in the blockchain literature.

\subsection{Improving privacy}

In the blockchain, every block and its associated data is propagated to and stored on every node of a chain in a P2P network. Each node of the blockchain then must be able 
to validate and execute each transaction, so transactions cannot be encrypted to maintain confidentiality, which eventually leads to privacy leakage. This type of privacy leakage may result in a breach of transaction information confidentiality. Confidentiality and privacy, therefore, pose a major challenge to blockchain and its applications, including sensitive transactions and private information (Zhang et al., 2019). To avoid such a scenario, accounting firms should utilise permissioned blockchain technology instead of a permissionless blockchain. A permissioned blockchain refers to a type of blockchain with restrictions in its membership and control procedures, which entails properties such as trade privacy, preservation, and noninvolvement. In such blockchain systems, the configuration intrinsically defines the role played by participants in which certain members can access, authorise new members in the system, and record the information on the blockchain (Liu et al., 2019). Appropriate deployment of access-control layers in this type of blockchain has greater potential to maintain privacy. Mitani and Otsuka (2020) suggests that privacy protection and high transparency are achievable by modelling traceability based on the hidden Markov model. The model is encrypted with homomorphic encryption, and information is verified by applying the non-interactive zero-knowledge proof of the knowledge that the plaintext is equal to zero.

\subsection{Regulatory issues}

The surge of blockchain cases around the world has attracted regulators in the USA, China, Japan, South Korea, Switzerland, Canada, UK, Australia, Singapore, Hong Kong, among others to regulate blockchain and protect consumers from illegal activities and fraud (Michael et al., 2018). However, regulatory challenges are manifold and will not only have a profound impact on existing regulations or even the policymaker 's current thinking, but they may also have to change their attitude towards technology. As technology is novel, the concepts around this technology, such as smart contracts and distributed ledgers, are different types of legal objects and can conflict with an existing legal framework, as traditional concepts of jurisdiction, liability, or compliance do not easily fit. Dealing with blockchain will probably require re-defining certain fundamental principles of legislation and re-defining lawyers, policymakers, and regulators (Cermeño, 2016). Michael et al. (2018) argues that to avoid a regulatory investigation, blockchain.based organisations must be willing to demonstrate the compliance of blockchain networks with industry regulations and guidelines. Michael et al. (2018) also suggests that some of the agencies that are likely to investigate and regulate blockchain systems are Securities and Exchange Commission (SEC), Commodities and Futures Trading Commission (CFTC), and Financial Institution Regulatory Authority (FINRA).

\section{Conclusions}

This paper introduced blockchain technology and its impact on accounting in general and pointed out some future directions. The advent of new blockchain technologies is not only helping businesses to transform their everyday operation, but also it is radically improving the efficacy of their processes. However, in the case of accounting industries, it still relies on traditional double-entry bookkeeping information systems. In our review, we found that there is ongoing research regarding the triple-entry 
bookkeeping information systems. Nevertheless, our finding suggests that even though the triple-entry system is desirable, it is not tamper-proof like blockchain. For instance, the trusted third party can never guarantee that the transaction between two parties will be safe from attacks or misuse; this is one of the accounting firm's major concerns. We believe that the adoption of blockchain technology will ultimately resolve this problem. In addition to the fact that information and data availability in real-time have a beneficial effect on business every day, it will also significantly help regulators make decisions. Contrary to the traditional financial practice, which is often erroneous and fraudulent, features that blockchain technology showcases cannot be ignored, such as smart contracts, transactional immutability, etc. Given that this technology is at its early stages of development, there are several challenges. One major technical challenge that's impeding the development of this technology is scalability.

Undoubtedly, blockchain improves the accounting profession by reducing the cost of maintaining and reconciling ledgers. Nevertheless, blockchain is seen as a substitute for bookkeeping, and reconciliation could be seen as a threat to accounting professionals. This could have a considerable negative impact on widespread adoption in the accounting domain. It is safe to say that there are still many unknowns concerning how blockchain will impact the audit and assurance profession, including the speed with which it will do so, as the technology has not reached its maturity yet. Furthermore, regulatory uncertainty around the technology has contributed negatively towards its acceptance.

If this technology is fully embraced, then the accounting business model's paradigm will surely alter radically. Also, we assume that the blockchain reinforced with a privacy-preserving deep learning system will make the audit process much smoother and error-free. The skill spectrum of the accountancy profession, however, will change to IT skills. At the current pace, blockchain technology will take years to reach its maturity. Either blockchain technology will thrive in the accounting domain or some sort of distributed ledger technology will appear in the future because the reward of reduced reconciliation, accurate information, real-time data, and the tamper-proof transaction is indeed too good to ignore.

\section{Acknowledgements}

The work was partially supported by National Science Foundation grants \#2000348, \#1761735, \#1723586, and \#1663350. Any opinions, findings, and conclusions or recommendations expressed in this material are those of the authors and do not necessarily reflect the views of the funding agencies.

\section{References}

Alladi, T., Chamola, V., Parizi, R.M. and Choo, K-K.R. (2019) 'Blockchain applications for Industry 4.0 and industrial IoT: a review', IEEE Access, IEEE, Vol. 7, pp.176935-176951.

Appelbaum, D. and Smith, S.S. (2018) 'Blockchain basics and hands-on guidance: taking the next step toward implementation and adoption', The CPA Journal, Vol. 88, No. 6, pp.28-37, New York State Society of Certified Public Accountants.

Baliga, A. (2017) 'Understanding blockchain consensus models', Persistent, Vol. 4, pp.1-14. 
Beigel, O. (2020) Who Accepts Bitcoin as Payment [online] https://99bitcoins.com/bitcoin/ who-accepts/ (accessed 29 May 2020).

Bible, W., Raphael, J., Taylor, P. and Valiente, I.O. (2017), Blockchain Technology and Its Potential Impact on the Audit and Assurance Profession [online] http://aicpa.org.

Blocksplain (2018) The Blockchain Scalability Problem \& The Race for Visa-Like Transaction Speed [online] https://towardsdatascience.com/the-blockchain-scalability-problem-the-race-for-visa-liketransaction-speed-5cce48f9d44 (accessed 4 April 2020).

Brakeville, S. and Bhargav, P. (2016) Blockchain Basics: Introduction to Business Ledgers, Issued by IBM Corporation.

Casino, F., Dasaklis, T.K. and Patsakis, C. (2019) 'A systematic literature review of blockchain-based applications: current status, classification and open issues', Telematics and Informatics, Vol. 36, pp.55-81, Elsevier.

Castillo, D.M. and Schifrin, M. (2020) Forbes Blockchain 50 [online] https://www.forbes.com/sites/ michaeldelcastillo/2020/02/19/blockchain-50/\#49b761827553 (accessed 6 June 2020).

Cermeño, J.S. (2016) 'Blockchain in financial services: regulatory landscape and future challenges for its commercial application', BBVA Research Paper, Vol. 16, p.20.

Chauhan, A., Malviya, O.P., Verma, M. and Mor, T.S. (2018) 'Blockchain and scalability', in The Proceedings of IEEE International Conference on Software Quality, Reliability and Security Companion (QRS-C) (2018), pp.122-128.

Christensson, P. (2018) Blockchain Definition [online] https://techterms.com/definition/blockchain (accessed 6 June 2020).

Das, S. (2017) Big 4 Accounting Giant PwC Accepts its First Bitcoin Payment [online] https://www. ccn.com/big-4-accounting-giant-pwc-accepts-first-bitcoin-payment/ (accessed 9 June 2020).

Delliott Insights (2019) Delliott's 2019 Global Blockchain Survey [online] https://www2.deloitte.com/ content/dam/Deloitte/se/Documents/risk/DI_2019-global-blockchain-survey.pdf (accessed 4 May 2020).

Dinh, T.N. and Thai, M.T. (2018) 'AI and blockchain: a disruptive integration', The CPA Journal, IEEE Computer, Vol. 51, No. 9, pp.48-53.

EY (2018) EY Announces Blockchain Audit Technology?, EY [online] https://www.ey.com/en_no/ news/2018/04/ey-announces-blockchain-audit-technology (accessed 6 June 2020).

Gramoli, V. and Staples, M. (2018) 'Blockchain standard: can we reach consensus?', IEEE Communications Standards Magazine, IEEE, Vol. 2, No. 3, pp.16-21.

Grigg, I. (2005) Triple Entry Accounting, Systemics Inc.

Gupta, Y., Shorey, R., Kulkarni, D. and Tew, J. (2018) 'The applicability of blockchain in the internet of things', in The Proceedings of 10th International Conference on Communication Systems \& Networks (COMSNETS), pp.561-564.

Han, M., Li, Z., He, J., Wu, D., Xie, Y. and Baba, A. (2018) 'A novel blockchain-based education records verification solution', in Proceedings of the 19th Annual SIG Conference on Information Technology Education, pp.178-183.

Hayes, A. (2020) Scalability, Investopedia [online] https://www.investopedia.com/terms/s/scalability. asp (accessed 25 March 2020).

Henry, R., Herzberg, A. and Kate, A. (2018) 'Blockchain access privacy: challenges and directions', IEEE Security \& Privacy, IEEE, Vol. 16, No. 4, pp.38-45.

Institute of Chartered Accountants in England and Wales (ICAEW) (2017) Blockchain and the Future of Accountancy [online] https://www.icaew.com/-/media/corporate/files/technical/ information-technology/thought-leadership/blockchain-and-the-future-of-accountancy.ashx (accessed 15 March 2020).

Inghirami, I.E. (2020) 'Accounting information systems: the scope of blockchain accounting', Digital Business Transformation, pp.7-120, Springer. 
Intuit (2020) Role-based Access Control for CFOs \& Accounting Departments [online] https://quickbooks.intuit.com/r/growing-complex-businesses/role-based-access-control-for-cfosaccounting-departments/ (accessed 14 June 2020).

Johnson, C. (2020) Google Goes Blockchain - New Deal Opens A Door To Crypto, Forbes [online] https://www.forbes.com/sites/coryjohnson/2020/05/27/google-goes-blockchain/ \#6d395a9b6593 (accessed 6 June 2020).

Khan, M., Quasim, M., Algarni, F. and Alharthi, A. (2020) Decentralised Internet of Things A Blockchain Perspective: A Blockchain Perspective, Springer Nature, Switzerland.

Khan, N. (2017) Demystifying Blockchain for Islamic Finance, Linkedin [online] https://www.linkedin. com/pulse/demystifying-blockchain-islamic-finance-nida-khan (accessed 8 June 2020).

Kim, H., Kim, S-H., Hwang, J.Y. and Seo, C. (2019) 'Efficient privacy-preserving machine learning for blockchain network', IEEE Access, IEEE, Vol. 7, pp.136481-136495.

Konstantinos, C. and Michael D. (2016) 'Blockchains and smart contracts for the internet of things', IEEE Access, Vol. 4, pp.2292-2303.

Kosba, A., Miller, A., Shi, E., Wen, Z. and Papamanthou, C. (2016) 'Hawk: the blockchain model of cryptography and privacy-preserving smart contracts', in The Proceedings of IEEE Symposium on Security and Privacy (SP), pp.839-858.

Leewayhertz (2020) A Beginner's Guide to Blockchain as a Service [online] https://https://www. leewayhertz.com/guide-to-blockchain-as-a-service/ (accessed 12 November 2020).

Li (2019) Blockchain Speeds \& The Scalability Debate [online] https://blocksplain.com/2018/02/28/ transaction-speeds/ (accessed 6 June 2020).

Liu, M., Wu, K. and Xu, J.J. (2019) 'How will blockchain technology impact auditing and accounting: permissionless versus permissioned blockchain', Current Issues in Auditing, Vol. 13, pp.A19-A29, American Accounting Association.

Lu, Y. (2018) 'Blockchain: a survey on functions, applications and open issues', Journal of Industrial Integration and Management, Vol. 3, No. 4, p.1850015, World Scientific.

Michael, J., Cohn, A. and Butcher, J.R. (2018) 'Blockchain technology', IEEE Spectrum, Vol. 1, No. 7.

Mitani, T. and Otsuka, A. (2020) 'Traceability in permissioned blockchain', IEEE Access, IEEE, Vol. 8, pp.21573-21588.

Nakamoto, S. (2008) Bitcoin: A Peer-to-Peer Electronic Cash System [online] https://bitcoin.org/ bitcoin.pdf (accessed 15 February 2020).

Nguyen, G-T. and Kim, K. (2018) 'A survey about consensus algorithms used in blockchain', Journal of Information Processing Systems, Vol. 14.

O’Neal, S. (2019) Big Four and Blockchain: Are Auditing Giants Adopting Yet? [online] https: //cointelegraph.com/news/big-four-and-blockchain-are-auditing-giants-adopting-yet (accessed 9 June 2020) .

Pacy, E.P. (2014) 'Tales from cryptocurrency: on Biocoin, Square Pegs, and Round Holes', New Eng. L. Rev., HeinOnline, Vol. 49, p.121.

Peck, M.E. (2017) 'Blockchains: how they work and why they'll change the world', IEEE Spectrum, IEEE, Vol. 54, pp.26-35.

Perez Yessi, B. (2019) KPMG Survey: 67\% of Corporates Are Not Using Blockchain Technology [online] https://thenextweb.com/hardfork/2019/04/09/kpmg-survey-67-of-corporates-are-not-usingblockchain-technology/ (accessed 4 June 2020).

Puthal, D., Malik, N., Mohanty, P.S., Kougianos, E. and Das, G. (2018) 'Everything you wanted to know about the blockchain: its promise, components, processes, and problems', IEEE Consumer Electronics Magazine, IEEE, Vol. 17, pp.6-14.

PwC (2018) Blockchain is Here. What's Your Next Move?, PwC [online] https://www.pwc.com/ blockchainsurvey (accessed 5 June 2020). 
Rückeshäuser, N. (2017) 'Do we really want blockchain-based accounting?', Decentralized Consensus as Enabler of Management Override of Internal Controls, Wirtschaftsinformatik und Angewandte Informatik.

Raikwar, M., Gligoroski, D. and Kralevska, K. (2019) 'Sok of used cryptography in blockchain', IEEE Access, Vol. 7, pp.148550-148575.

Salimitari, M., Joneidi, M. and Chatterjee, M. (2019) 'AI-enabled blockchain: an outlier-aware consensus protocol for blockchain-based IoT networks', 2019 IEEE Global Communications Conference (GLOBECOM), IEEE, Vol. 7, pp.1-6.

Singh, J. and Michels, J.D. (2018) 'Blockchain as a service (BaaS): providers and trust', 2018 IEEE European Symposium on Security and Privacy Workshops (EuroS\&PW), IEEE, pp.67-74.

Sompolinsky, Y. and Zohar, A. (2018) PHANTOM, GHOSTDAG: Two Scalable BlockDAG Protocols, IACR Cryptology ePrint Archive.

Stanley, A. (2018) PwC: Regulatory Uncertainty is Largest Impediment to Blockchain Adoption [online] https://www.forbes.com/sites/astanley/2018/08/27/pwc-regulatory-uncertainty-is-largestimpediment-to-blockchain-adoption/\#6037dcf0b0144 (accessed 7 June 2020).

Swan, M. (2015) Blockchain: Blueprint for a New Economy, O’Reilly Media, Inc.

Taskinsoy, J. (2019) 'A misunderstood digital revolution', Things You Need to Know about Blockchain.

The Economist (2015) The Great Chain of Being Sure About Things, The Economist [online] https://www.economist.com/briefing/2015/10/31/the-great-chain-of-being-sure-about-things (accessed 15 March 2020).

Vetter, A. (2018) Voices Blockchain is Already Changing Accounting [online] https://www. accountingtoday.com/opinion/blockchain-is-already-changing-accounting (accessed 9 June 2020).

Vukolić, M. (2015) 'The quest for scalable blockchain fabric: proof-of-work vs. BFT replication', in The Proceedings of International Workshop on Open Problems in Network Security, Springer, pp.112-125.

Zamani, M., Mahnush, M. and Raykova, M. (2018) 'RapidChain: scaling blockchain via full sharding', in The Proceedings of the ACM SIGSAC Conference on Computer and Communications Security (CCS'18), pp.931-948.

Zhang, R., Xue, R. and Liu, L. (2019) 'Security and privacy on blockchain', ACM Computing Surveys (CSUR), Vol. 52, pp.1-34, ACM New York, NY, USA.

Zheng, Z., Xie, S., Dai, H., Chen, X. and Wang, H. (2017) 'An overview of blockchain technology: architecture, consensus, and future trends', in The Proceedings of IEEE International Congress on Big Data (BigData Congress), pp.557-564.

Zheng, Z., Xie, S., Dai, H-N., Chen, X. and Wang, H. (2018) 'Blockchain challenges and opportunities: a survey', International Journal of Web and Grid Services, Vol. 14, No. 4, pp.352-375, Inderscience Publishers (IEL), DOI: 10.1504/IJWGS.2018.095647. 